Bull. Austral. Math. Soc.

$34 \mathrm{~A} 12,34 \mathrm{~K} 30,47 \mathrm{D} 06,34 \mathrm{~A} 30$

VoL. 69 (2004) [383-394]

\title{
ON THE $n$-PARAMETER ABSTRACT CAUCHY PROBLEM
}

\author{
M. JANFADA AND A. NiKNAM
}

Let $H_{i}(i=1,2, \ldots, n)$, be closed operators in a Banach space $X$. The generalised initial value problem

$$
\left\{\begin{array}{l}
\frac{\partial}{\partial t_{i}} u\left(t_{1}, t_{2}, \ldots, t_{i}, \ldots, t_{n}\right)=H_{i} u\left(t_{1}, \ldots, t_{n}\right), \quad t_{i} \in\left(0, T_{i}\right] \quad i=1,2, \ldots, n \\
u(0)=x, \quad x \in \bigcap_{i=1}^{n} D\left(H_{i}\right),
\end{array}\right.
$$

of the abstract Cauchy problem is studied. We show that the uniqueness of solution $u:\left[0, T_{1}\right] \times\left[0, T_{2}\right] \times \cdots \times\left[0, T_{n}\right] \rightarrow X$ of this $n$-abstract Cauchy problem is closely related to $C_{0}-n$-parameter semigroups of bounded linear operators on $X$. Also as another application of $C_{0}$ - $n$-parameter semigroups, we prove that many $n$-parameter initial value problems cannot have a unique solution for some initial values.

\section{INTRODUCTION}

Suppose $X$ is a Banach space and $A$ is a linear operator from $D(A) \subseteq X$ into $X$. Given $x \in X$, the abstract Cauchy problem for $A$ with the initial value $x$, consists of finding a solution $u(t)$ to the initial value problem

$$
\left\{\begin{array}{l}
\frac{d u(t)}{d t}=A u(t) \quad t \in(0, T] \\
u(0)=x
\end{array}\right.
$$

where by a solution we mean an $X$-valued function $u:[0, T] \rightarrow X$ which is continuous for $t \geqslant 0$, continuously differentiable for $t>0, u(t) \in D(A)$ for $t \in(0, T]$ and (1) is satisfied.

A one-parameter semigroup of operators is a homomorphism $T:\left(\mathbb{R}_{+},+\right) \rightarrow B(X)$ for which $T(0)=I$, where $\mathbb{R}_{+}=[0, \infty)$ and $B(X)$ is the Banach space of all bounded linear operators on $X$. The one-parameter semigroup $\{T(t)\}_{t \geqslant 0}$ is called strongly continuous (or $C_{0}$-continuous) if $\lim _{t \rightarrow 0} T(t) x=x$, for each $x \in X$ and is called uniformly continuous if $\lim _{t \rightarrow 0} T(t)=I$ in $B(X)$. The linear mapping $A$ defined by

$$
A(x)=\lim _{t \rightarrow 0} \frac{T(t) x-x}{t}
$$

\section{Received 22nd May, 2003}

This work has been done under supervision of Professor J. Phillips when the first author has been in University of Victoria for a six month visit.

Copyright Clearance Centre, Inc. Serial-fee code: 0004-9727/04 \$A2.00+0.00. 
where $D(A)=\left\{x: \lim _{t \rightarrow 0}(T(t) x-x) / t\right.$ exist $\}$, is called the infinitesimal generator of $\left(T, \mathbb{R}_{+}, X\right)$.

The following Theorem which is due to Hille [5], shows the close relation of abstract Cauchy problem with semigroup theory (see also [4]).

ThEOREM 1.1. Let $A$ be a closed linear operator in Banach space $X$, then the following are equivalent:

(a) For each $x \in D(A)$ there exists a unique solution for (1).

(b) The part $A_{1}=A_{\left.\right|_{1}}$ of $A$ in $X_{1}:=\left(D(A),\|\cdot\|_{A}\right)$ is the infinitesimal generator of a $C_{0}$-one-parameter semigroup of operators on the Banach space $X_{1}$, where $\|\cdot\|_{A}$ is the graph norm on $D(A)$.

PROOF: [2, II.6.6].

The previous Theorem has many applications in inhomogenous initial value problems and evaluation systems. One can see some more applications of abstract Cauchy problem in $[3,7]$.

Let $\mathbb{R}_{+}^{n}=\left\{\left(t_{1}, t_{2}, \ldots, t_{n}\right): t_{i} \geqslant 0, i=1,2, \ldots, n\right\}$. By an $n$-parameter semigroup of operators we mean a homomorphism $W:\left(\mathbb{R}_{+}^{n},+\right) \rightarrow B(X)$ for which $W(0)=I$ and denote it by $\left(W, \mathbb{R}_{+}^{n}, X\right)$. Suppose $H_{i}$ is the infinitesimal generator of the one-parameter semigroup $\left\{W\left(t e_{i}\right)\right\}_{t \geqslant 0}$, where $\left\{e_{i}\right\}_{i=1}^{n}$ is the standard basis of $\mathbb{R}^{n}$, we shall think of $\left(H_{1}, H_{2}, \ldots, H_{n}\right)$ as the infinitesimal generator of $W$. As in the oneparameter case, $\left(W, \mathbb{R}_{+}^{n}, X\right)$ is called strongly continuous (or $C_{0}$-continuous ) if for each $x \in X, \lim _{t \rightarrow 0} W(t) x=x$, and is called uniformly continuous if $\lim _{t \rightarrow 0} W(t)=I$, where $t \rightarrow 0$ in $\mathbb{R}_{+}^{n}$. It is not hard to see that $\left(W, \mathbb{R}_{+}^{n}, X\right)$ is a $C_{0}$-continuous (respectively uniformly continuous) if and only if for each $i=1,2, \ldots, n,\left\{W\left(t e_{i}\right)\right\}_{t \geqslant 0}$ is strongly (respectively uniformly) continuous. The following useful proposition which states some basic Properties of $n$-parameter semigroups can be found in [1] as is described in [6].

PROPOSITION 1.2. Suppose $\left(W, \mathbb{R}_{+}^{n}, X\right)$ is a $C_{0}$ - $n$-parameter semigroup then

(a) If $x \in D\left(H_{i}\right)$, so does $W(t) x$, for each $t \in \mathbb{R}_{+}^{n}$ and

$$
H_{i} W(t) x=W(t) H_{i} x \quad(i=1,2, \ldots, n) .
$$

(b) $\bigcap_{i=1}^{n} D\left(H_{i}\right)$ is dense in $X$, and $X_{1}=\left(\bigcap_{i=1}^{n} D\left(H_{i}\right),\|\cdot\|_{1}\right)$ is a Banach space, where for $x \in \bigcap_{i=1}^{n} D\left(H_{i}\right),\|x\|_{1}=\|x\|+\sum_{i=1}^{n}\left\|H_{i}(x)\right\|$.

(c) For each $1 \leqslant i, j \leqslant n, D\left(H_{i}\right) \cap D\left(H_{i} H_{j}\right) \subseteq D\left(H_{j} H_{i}\right)$, and for $x \in D\left(H_{i}\right) \cap D\left(H_{i} H_{j}\right)$,

$$
H_{i} H_{j}(x)=H_{j} H_{i}(x)
$$

In the rest of this note we shall state an extension of one-parameter abstract Cauchy problem and establish its relation with $C_{0}$-n-parameter semigroups of operators. As 
another application of $C_{0}$ - $n$-parameter semigroups we shall show that some $n$-parameter initial valued problems cannot have a unique solution. The abstract Cauchy problem also admits another natural generalisation which is discussed in $[5,6,8]$.

\section{The MAin RESUlts}

Suppose as before $X$ is a Banach space, $H_{i}$ are closed linear operators from $D\left(H_{i}\right)$ $\subseteq X$ into $X$ and $T_{i}>0,(i=1,2, \ldots, n)$. Then, a continuous $X$-valued function $u:\left[0, T_{1}\right] \times \cdots \times\left[0, T_{n}\right] \rightarrow X$ with continuous partial derivatives which satisfy the following $n$-parameter abstract Cauchy problem ( $n$-abstract Cauchy problem)

$$
\left\{\begin{array}{l}
\frac{\partial}{\partial t_{i}} u\left(t_{1}, t_{2}, \ldots, t_{i}, \ldots, t_{n}\right)=H_{i} u\left(t_{1}, \ldots, t_{n}\right), \quad i=1,2, \ldots, n \quad t_{i} \in\left(0, T_{i}\right] \\
u(0)=x, \quad x \in \bigcap_{i=1}^{n} D\left(H_{i}\right),
\end{array}\right.
$$

is called a solution of the initial value problem (2).

For convenience in the rest of this note we denote by $I_{T}$ the positive $n$-cell $\left[0, T_{1}\right] \times\left[0, T_{2}\right] \times \cdots \times\left[0, T_{n}\right]$ where $T=\left(T_{1}, T_{2}, \ldots, T_{n}\right) \in \mathbb{R}_{+}^{n}$ and $T_{i}>0$. As mentioned in the previous section, we shall illustrate that (2) is closely related to $C_{0}-n$ parameter semigroups of operators. In the following theorem we prove that if $I_{T}$ is arbitrary and $\left(H_{1}, H_{2}, \ldots, H_{n}\right)$ is the infinitesimal generator of a $C_{0}-n$-parameter semigroup $\left(W, \mathbb{R}_{+}^{n}, X\right)$, then (2) has the unique solution $u\left(t_{1}, t_{2}, \ldots, t_{n}\right)=W\left(t_{1}, t_{2}, \ldots, t_{n}\right) x$, for each $x \in \bigcap_{i=1}^{n} D\left(H_{i}\right)$, where $\left(t_{1}, t_{2}, \ldots, t_{n}\right) \in I_{T}$.

THEOREM 2.1. Suppose $I_{T}$ is a positive $n$-cell corresponding to $T \in \mathbb{R}_{+}^{n}$, and $\left(H_{1}, H_{2}, \ldots, H_{n}\right)$ is the infinitesimal generator of the $C_{0}$-n-parameter semigroup $\left(W, \mathbb{R}_{+}^{n}, X\right)$ of operators, then for each $x \in \bigcap_{i=1}^{n} D\left(H_{i}\right)$ the $n$-abastract Cauchy problem (2) has a unique solution.

Proof: Let $I_{T}$ be arbitrary, $\left\{e_{i}\right\}_{i=1}^{n}$ be the standard basis of $\mathbb{R}^{n}$ and $H_{i}$ be the infinitesimal generator of the $C_{0}-n$-parameter semigroup $\left\{W\left(t_{i}\right)\right\}_{i \geqslant 0}$. For $x \in \bigcap_{i=1}^{n} D\left(H_{i}\right)$, define $u: I_{T} \rightarrow X$ by $u(t)=W(t) x$. One can easily see that $u(t)$ is a solution of $n$-abstract Cauchy problem (2) for the initial value $x \in \bigcap_{i=1}^{n} D\left(H_{i}\right)$. For proving the uniqueness of solution it is enough to show that (2) has no proper (that is, nonzero) solution for the initial value $x=0$. Theorem 1.1 shows that for each $i=1,2, \ldots, n$, the initial value problem

$$
\begin{cases}\frac{d u^{i}(s)}{d s}=H_{i} u^{i}(s) & s \in\left(0, T_{i}\right] \\ u^{i}(0)=x & x \in D\left(H_{i}\right)\end{cases}
$$


has a unique solution for each $x \in D\left(H_{i}\right)$. By definition of solution we know that for $t \in I_{T}, u(t)$ which is a solution of (2) for $x=0$, is in $\bigcap_{i=1}^{n} D\left(H_{i}\right)$, so for the initial value $x=u\left(t_{1}, t_{2}, \ldots, t_{i-1}, 0, t_{i+1}, \ldots, t_{n}\right) \in D\left(H_{i}\right), u^{i}(s)=u\left(t_{1}, \ldots, t_{i-1}, s, t_{i+1}, \ldots, t_{n}\right)$ and $v^{i}(s)=W\left(s e_{i}\right) u\left(t_{1}, \ldots, t_{i-1}, 0, t_{i+1}, \ldots, t_{n}\right)$ is a solution of $(3)$ for $\mathbf{x}$. Uniqueness of solution of (3) implies that

$$
\begin{aligned}
W\left(s e_{i}\right) u\left(t_{1}, \ldots, t_{i-1}, 0, t_{i+1}, \ldots, t_{n}\right) & =v^{i}(s) \\
& =u^{i}(s)=u\left(t_{1}, \ldots, t_{i-1}, s, t_{i+1}, \ldots, t_{n}\right),
\end{aligned}
$$

for each $i=1,2, \ldots, n, 0 \leqslant s \leqslant T_{i}$ and $0 \leqslant t_{j} \leqslant T_{j}, i \neq j=1,2, \ldots, n$. Using (4) for $t=\sum_{i=1}^{n} t_{i} e_{i} \in I_{T}$, shows that

$$
\begin{array}{rlrl}
u(t)=u\left(t_{1}, t_{2}, \ldots, t_{n}\right) & =W\left(t_{1} e_{1}\right) u\left(0, t_{2}, \ldots, t_{n}\right) & \left(i=1, s=t_{1}\right) \\
& =W\left(t_{1} e_{1}\right)\left(W\left(t_{2} e_{2}\right) u\left(0,0, t_{3}, \ldots, t_{n}\right)\right. & \left(i=2, s=t_{2}\right) \\
(W \text { in } n \text {-parameter }) & =W\left(\sum_{i=1}^{n} t_{i} e_{i}\right) u(0,0, \ldots, 0)=W(t)(0)=0
\end{array}
$$

Hence $u(t)=0$ and (2) cannot have a proper solution for $x=0$, or equivalently (2) has a unique solution for each $x \in \bigcap_{i=1}^{n} D\left(H_{i}\right)$.

Now let $H_{i}$ 's $(i=1,2, \ldots, n)$ from $D\left(H_{i}\right) \subseteq X$ into $X$ be closed operators. Similarly to Proposition 1.2 (b) one can see $X_{1}=\left(\bigcap_{i=1}^{n} D\left(H_{i}\right),\|\cdot\|_{1}\right)$, where $\|x\|_{1}$ $=\|x\|+\sum_{i=1}^{n}\left\|H_{i}(x)\right\|, \quad\left(x \in \bigcap_{i=1}^{n} D\left(H_{i}\right)\right)$ is a Banach space. In the next theorem we are going to show that for positive $n$-cells $I_{T}$ and $I_{T^{\prime}}$, where $I_{T} \subseteq I_{T^{\prime}}$, if (2) has a unique solution for each $x \in X_{1}$ then there exist a $C_{0}$ - $n$-parameter semigroup $\left(W, \mathbb{R}_{+}^{n}, X_{1}\right)$ with the infinitesimal generator $\left(K_{1}, K_{2}, \ldots, K_{n}\right)$ for which $W(t) x=u(t ; x)$, the unique solution of (2) for $x \in X_{1}$ and $t \in I_{T}$, also for $x \in D\left(K_{i}\right), K_{i}(x)=H_{i}(x)$.

TheOREM 2.2. Suppose $H_{i}$ 's $(i=1,2, \ldots, n)$ are closed linear operators and for positive $n$-cells $I_{T}$ and $I_{T^{\prime}}$, where $I_{T} \subseteq I_{T^{\prime}}$, the $n$-abstract Cauchy problem (2) has a unique solution for each $x \in X_{1}$, then there exist a $C_{0}$-n-parameter semigroup $\left(W, \mathbb{R}_{+}^{n}, X_{1}\right)$ of linear bounded operators with the infinitesimal generator $\left(K_{1}, K_{2}, \ldots, K_{n}\right)$ such that for $t \in I_{T}$ and $x \in X_{1}, W(t) x=u(t ; x)$ where $u(t ; x)$ is the unique solution of (2) for the initial value $x$, and for $x \in D\left(K_{i}\right), K_{i}(x)=H_{i}(x)$.

Proof: Let $u(t ; x)$ be the unique solution of (2) for $x \in X_{1}$. For $t \in I_{T}$, we define the operator $W_{1}(t): X_{1} \rightarrow X_{1}$ by $W_{1}(t) x=u(t ; x)$. Trivially $W_{1}(t)$ is well-defined and a linear operator, since the solution is unique. We are going to show that $W_{1}(t)$ is bounded. Define the mapping $\Phi: X_{1} \rightarrow C^{1}\left(I_{T}, X_{1}\right)$ by $\Phi(x)(t)=W_{1}(t)(x)$, where $C^{1}\left(I_{T}, X_{1}\right)$ is the Banach space of all continuous $X_{1}$-valued functions on $I_{T}$ with continuous partial 
derivative, equiped with the supremum norm. $\Phi$ is linear, we prove it is closed. Suppose $x_{m} \rightarrow x$ in $X_{1}$ and $\Phi\left(x_{m}\right) \rightarrow f$ in $C^{1}\left(I_{T}, X_{1}\right)$, integrating of (2) implies that for each $i=1,2, \ldots, n, m \in \mathbb{N}$ and $t=\left(t_{1}, \ldots, t_{n}\right) \in I_{T}$,

$$
\begin{aligned}
W_{1}\left(t_{1}, \ldots, t_{n}\right) x_{m}=W_{1}\left(t_{1}, t_{2}, \ldots,\right. & \left.t_{i-1}, 0, t_{i+1}, \ldots, t_{n}\right) x_{m} \\
& +\int_{0}^{t_{i}} H_{i} W_{1}\left(t_{1}, t_{2}, \ldots, t_{i-1}, s, t_{i+1}, \ldots, t_{n}\right) x_{m} d s .
\end{aligned}
$$

Let $m \rightarrow \infty$, so $\operatorname{Sup}_{t \in I_{T}}\left\|\Phi\left(x_{m}\right)(t)-f(t)\right\|_{1} \rightarrow 0$, this, (5), together with the closedness of $H_{i}$, imply that for each $i=1,2, \ldots, n$,

$$
\begin{aligned}
f\left(t_{1}, \ldots, t_{n}\right)=f\left(t_{1}, t_{2}, \ldots, t_{i-1}, 0, t_{i+1}, \ldots, t_{n}\right) & \\
& +\int_{0}^{t_{i}} H_{i} f\left(t_{1}, t_{2}, \ldots, t_{i-1}, s, t_{i+1}, \ldots, t_{n}\right) d s .
\end{aligned}
$$

Thus (6) and the fact that $f \in C^{1}\left(I_{T}, X_{1}\right)$ show that

$$
\left\{\begin{array}{l}
\frac{\partial}{\partial t_{i}} f\left(t_{1}, t_{2}, \ldots, t_{i}, \ldots, t_{n}\right)=H_{i} f\left(t_{1}, \ldots, t_{n}\right), \quad i=1,2, \ldots, n \quad t_{i} \in\left(0, T_{i}\right] \\
f(0)=\lim _{m \rightarrow \infty} \Phi\left(x_{m}\right)(0)=\lim _{m \rightarrow \infty} W_{1}(0)\left(x_{m}\right)=x .
\end{array}\right.
$$

Hence $f$ is a solution of (2) for the initial value $x$, the uniqueness of solution gives

$$
f(t)=W_{1}(t) x=\Phi(x) t, \quad t \in I_{T}
$$

it means that $\Phi$ is closed operator from the Banach space $X_{1}$ into the Banach space $C^{1}\left(I_{T}, X_{1}\right)$ and the closed graph theorem tell us

$$
\operatorname{Sup}_{\|x\|_{1} \leqslant 1}\left\|W_{1}(\cdot) x\right\|_{\infty}=\operatorname{Sup}_{\|x\|_{1} \leqslant 1}\left(\operatorname{Sup}_{t \in I_{T}}\left\|W_{1}(t) x\right\|_{1}\right)=M<\infty .
$$

Thus for each $t \in I_{T}, W_{1}(t)$ is a bounded operator on $X_{1}$. Now let $T^{\prime \prime}$ $=\left(T_{1}^{\prime \prime}, T_{2}^{\prime \prime}, \ldots, T_{n}^{\prime \prime}\right)$, where $T_{i}^{\prime \prime}=\min \left\{T_{i}^{\prime}, T_{i}-T_{i}^{\prime}\right\}$. We are going to show that for each $t, t^{\prime} \in I_{T^{\prime \prime}}, W_{1}\left(t+t^{\prime}\right)=W_{1}(t) W_{1}\left(t^{\prime}\right)$. First we notice that for $t \in I_{T^{\prime}}$ and $t^{\prime} \in I_{T^{\prime \prime}}, t_{i} \leqslant T_{i}^{\prime}$ and $t_{i}^{\prime} \leqslant T_{i}-T_{i}^{\prime}$ so $t_{i}+t_{i}^{\prime} \leqslant T_{i}$ and $t+t^{\prime} \in I_{r}$. Let $t^{\prime}$ be fixed, for $x \in X_{1}$ define $v(\cdot): I_{T^{\prime}} \rightarrow X_{1}$ by $v(t)=W_{1}\left(t+t^{\prime}\right) x$. Trivially $v(t)$ and $u(t)=W_{1}(t) W_{1}\left(t^{\prime}\right) x$ are solutions of (2) in $I_{T^{\prime}}$ for the initial value $W_{1}\left(t^{\prime}\right) x$, by the uniqueness of solution of $(2)$ in $I_{T^{\prime}}$ we have

$$
W_{1}\left(t+t^{\prime}\right) x=v(t)=u(t)=W_{1}(t)\left(W_{1}\left(t^{\prime}\right) x\right) .
$$

Now we can extend $W_{1}$ to an $n$-parameter semigroup of operators. Let $s=\left(s_{1}, s_{2}, \ldots, s_{n}\right)$ $\in \mathbb{R}_{+}^{n}$, and choose $m_{i} \in \mathbb{N}$ and $r_{i} \in\left[0, T_{i}^{\prime \prime}\right]$ so that $s_{i}=m_{i} T_{i}^{\prime \prime}+r_{i}$, $i=1,2, \ldots, n$. Suppose

$$
W(s) x=W_{1}(r)\left[\Pi_{i=1}^{n}\left(W_{1}\left(T_{i}^{\prime \prime} e_{i}\right)\right)^{m_{i}}\right](x)
$$


where $r=\left(r_{1}, r_{2}, \ldots, r_{n}\right) \in I_{T^{\prime \prime}}$. By the previous parts of proof the operators in the right hand side of the last equality commute and are bounded linear operators on $X_{1}$. One can easily see that $\left(W, \mathbb{R}_{+}^{n}, X_{1}\right)$ is an $n$-parameter semigroup of operators and the fact that $\lim _{t \rightarrow 0} W(t) x=x$ (by continuity of $u(t ; x)$ ) show that $W$ is strongly continuous. Also for $s \in I_{T}, W_{1}(s) x=W(s) x$, since

$$
\begin{aligned}
\frac{\partial}{\partial s_{i}} W(s) x & =\frac{\partial}{\partial r_{i}} W_{1}(r)\left[\Pi_{i=1}^{n}\left(W_{1}\left(T_{i}^{\prime \prime} e_{i}\right)\right)^{m_{i}}\right](x) \\
& =H_{i} W_{1}(r)\left[\Pi_{i=1}^{n}\left(W_{1}\left(T_{i}^{\prime \prime} e_{i}\right)\right)^{m_{i}}\right](x)=H_{i} W(s) x
\end{aligned}
$$

and the equality holds from the uniqueness of solution in $I_{T}$. If $\left(K_{1}, K_{2}, \ldots, K_{n}\right)$ is the generator of $W$ and $x \in D\left(K_{i}\right) \subseteq X_{1}=\bigcap_{i=1}^{n} D\left(H_{i}\right)$, then

$$
\|\cdot\|_{1}-\lim _{t \rightarrow 0} \frac{W\left(t e_{i}\right) x-x}{t}=K_{i}(x)
$$

which implies $\lim _{t \rightarrow 0}\left(W\left(t e_{i}\right) x-x\right) / t=K_{i}(x)$, but $x \in D\left(H_{i}\right)$ and so

$$
\begin{aligned}
\lim _{t \rightarrow 0} \frac{W\left(t e_{i}\right) x-x}{t} & =\frac{\partial}{\partial t_{i}} W(0,0, \ldots, 0) x \\
& =H_{i} W(0) x=H_{i}(x)
\end{aligned}
$$

Thus $K_{i}(x)=H_{i}(x)$ and this complete the proof of theorem.

In the previous Theorem we could replace the assumption of existence of a unique solution for (2) in $I_{T}$ and $I_{T^{\prime}}$, by the assumption that (2) has a unique solution in $I_{T}$ and whole of $\mathbb{R}_{+}^{n}$, which seems stronger than our hypothesis. As another application of $C_{0}$-n-parameter semigroups, we shall show that for a closed linear operator $A: D(A)$ $\subseteq X \rightarrow X$, the $n$-parameter initial value problem

$$
\left\{\begin{array}{l}
\sum_{i=1}^{n} \frac{\partial}{\partial t i} u\left(t_{1}, t_{2}, \ldots, t_{n}\right)=A u\left(t_{1}, t_{2}, \ldots, t_{n}\right), \quad t=\left(t_{1}, t_{2}, \ldots, t_{n}\right) \in I_{T} \\
u(0)=x, \quad x \in D(A)
\end{array}\right.
$$

does not have a unique solution in both $I_{T}$ and $I_{T^{\prime}}$ for each $x \in D(A)$, for which $I_{T^{\prime}} \subseteq I_{T}$.

The initial value problem (7) can have a solution, for example if $\left(H_{1}, H_{2}, \ldots, H_{n}\right)$ is generator of a $C_{0}$ - $n$-parameter semigroup $\left(W, \mathbb{R}_{+}^{n}, X\right)$ and $A=H_{1}+H_{2}+\cdots+H_{n}$, then obviously $u(t)=W(t) x$ is a solution of $(7)$ in any positive $n$-cell $I_{T}$, for the initial value $x \in \bigcap_{i=1}^{n} D\left(H_{i}\right) \subseteq D(A)$

Before proving our claim we need the following lemmas.

LEMMA 2.3. Suppose $\{T(t)\}_{t \geqslant 0}$ is a $C_{0}$-one parameter semigroup of operators with the infinitesimal generator $A$, and $B \in B(X)$, then $A+B$ is the infinitesimal generator of a $C_{0}$-semigroup $S(t)$ on $X$ satisfying

$$
S(t) x=T(t) x+\int_{0}^{t} T(t-s) B S(s) x d s, \quad x \in X .
$$


PROOF: See [7, III.1.1 and III.1.2].

Also the next lemma which provide a necessary and sufficient condition for the composition of $C_{0}$-one parameter semigroups to be a $C_{0}$ - $n$-parameter semigroup, has a principal role in the next theorem.

Recall that for linear operator $H$ in Banach space $X, \rho(H)$ denotes the resorvent set of $H$ and for $\lambda \in \rho(H), R(\lambda ; H)$ is used for $(\lambda I-H)^{-1}$.

Lemma 2.4. Suppose $\left\{U^{i}(s)\right\}_{s \geqslant 0}$ is a $C_{0}$-one-parameter semigroup of operators on Banach space $X$ with the infinitesimal generator $H_{i},(i=1,2, \ldots, n)$, then $W\left(t_{1}, t_{2}, \ldots, t_{n}\right)=U^{1}\left(t_{1}\right) U^{2}\left(t_{2}\right) \ldots U^{n}\left(t_{n}\right)$ is a $C_{0}-n$-parameter semigroup of operators if and only if there is an $\omega>0$ such that for each $i=1,2, \ldots, n,[\omega, \infty) \subseteq \rho\left(H_{i}\right)$ and for each integers $0 \leqslant i, j \leqslant n$ and $\lambda, \lambda^{\prime} \geqslant \omega$, we have

$$
R\left(\lambda^{\prime} ; H_{j}\right) R\left(\lambda ; H_{i}\right)=R\left(\lambda ; H_{i}\right) R\left(\lambda^{\prime} ; H_{j}\right) .
$$

Proof: First suppose $W$ is a $C_{0}-n$-parameter semigroup of operators. Since $H_{i}$ is the infinitesimal generator of $\left\{u^{i}(t)\right\}_{t \geqslant 0}$, by the Hille-Yosida Theorem $([7,1.5 .3])$, there is an $\omega_{i}>0$ such that for each $\lambda \geqslant \omega_{i}, R\left(\lambda ; H_{i}\right)$ exist and are bounded operators. Let $\omega=\max \left\{\omega_{i}: i=1,2, \ldots, n\right\}$. If $\lambda \geqslant \omega$, from $[7$, I.5.4]

$$
R\left(\lambda ; H_{i}\right)(x)=\int_{0}^{\infty} e^{-\lambda s} U^{i}(s)(x) d s .
$$

Also we know that for each integers $0 \leqslant i, j \leqslant n$,

$$
U^{i}(s) U^{j}(t)=W\left(s e_{i}\right) W\left(t e_{j}\right)=W\left(t e_{j}\right) W\left(s e_{i}\right)=U^{j}(t) U^{i}(s),
$$

so

$$
\begin{aligned}
R\left(\lambda ; H_{i}\right)\left(U^{j}(t) x\right) & =\int_{0}^{\infty} e^{-\lambda t} U^{i}(s) U^{j}(t) x d s \\
& =\int_{0}^{\infty} e^{-\lambda t} U^{j}(t) U^{i}(s) x d s=U^{j}(t) \int_{0}^{\infty} e^{-\lambda t} U^{i}(s) x d s \\
& =u^{j}(t) R\left(\lambda ; H_{i}\right) x
\end{aligned}
$$

Now let $\lambda^{\prime} \geqslant \omega$, we know $R\left(\lambda ; H_{i}\right)$ is bounded so

$$
\begin{aligned}
R\left(\lambda ; H_{i}\right) R\left(\lambda^{\prime} ; H_{j}\right) x & =R\left(\lambda ; H_{i}\right) \int_{0}^{\infty} e^{-\lambda^{\prime} t} U^{j}(t) x d t \\
& =\int_{0}^{\infty} e^{-\lambda^{\prime} t} U^{j}(t) R\left(\lambda ; H_{i}\right) x d t \\
& =R\left(\lambda^{\prime} ; H_{j}\right) R\left(\lambda ; H_{i}\right) x
\end{aligned}
$$

and this prove the necessary part of lemma.

For the converse suppose there is an $\omega>0$ such that for each $\lambda, \lambda^{\prime}>0, R\left(\lambda ; H_{i}\right)$ and $R\left(\lambda^{\prime} ; H_{j}\right)$ exist and commute. So we have $H_{\lambda}^{i} H_{\lambda^{\prime}}^{j}=H_{\lambda^{\prime}}^{j} H_{\lambda}^{i}$ where $H_{\lambda}^{i}=\lambda^{2} R\left(\lambda ; H_{i}\right)-\lambda I$ 
and $H_{\lambda^{\prime}}^{j}=\lambda^{\prime 2} R\left(\lambda ; H_{j}\right)-\lambda^{\prime} I$ are the Yosida approximation of $H_{i}$ and $H_{j}$ respectively. Applying [7, I.3.5] we have $U^{i}(s) x=\lim _{\lambda \rightarrow \infty} e^{s H_{\lambda}^{i}} x$ and $U^{j}(t) x=\lim _{\lambda^{\prime} \rightarrow \infty} e^{t H_{\lambda^{\prime}}^{j} x \text {, thus }}$

$$
\begin{aligned}
& U^{i}(s) U^{j}(t) x=\lim _{\lambda \rightarrow \infty} e^{s H_{\lambda}^{i}} U^{j}(t) x \\
& =\lim _{\lambda \rightarrow \infty} e^{s H_{\lambda}^{i}}\left(\lim _{\lambda^{\prime} \rightarrow \infty} e^{t H_{\lambda^{\prime}}^{j} x}\right) \\
& =\lim _{\lambda \rightarrow \infty} \lim _{\lambda^{\prime} \rightarrow \infty} e^{s H_{\lambda}^{i}} e^{t H_{\lambda^{\prime}}^{j} x} \quad \text { (e } e^{s H_{\lambda}^{i}} \text { is continuous) }
\end{aligned}
$$

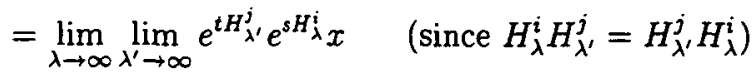

$$
\begin{aligned}
& =\lim _{\lambda \rightarrow \infty} U^{j}(t) e^{s H_{\lambda}^{i} x} \\
& =U^{j}(t) U^{i}(s) x \quad\left(U^{j}(t) \text { is continuous }\right) .
\end{aligned}
$$

Hence $W\left(t_{1}, t_{2}, \ldots, t_{n}\right)=U^{1}\left(t_{1}\right) U^{2}\left(t_{2}\right) \ldots U^{n}\left(t_{n}\right)$ is a $C_{0}$ - $n$-parameter semigroup of operators.

Now we are ready for this theorem.

TheOREM 2.5. Suppose $A$ is a closed operator from $D(A) \subseteq X$ into $X$ and $I_{T}$ and $I_{T^{\prime}}, I_{T^{\prime}} \subseteq I_{T}$, is given. Then the initial value problem (7) cannot have a unique solution for each $x \in D(A)$ in both $I_{T}$ and $I_{T^{\prime}}$.

Proof: Suppose to the contrary (7) has a unique solution for each $x \in D(A)$ in both $I_{T}$ and $I_{T^{\prime}}$. As in Theorem 2.2 we are going to show that if $u(t ; x)$ is the unique solution of (7) for $x \in D(A)$ and $t \in I_{T}$, then $W_{1}(t) x=u(t ; x)$ can be extended to a $C_{0}$ - $n$-parameter semigroup of operators, and using previous lemma we shall get a contradiction.

Obviously uniqueness of solution shows that $W_{1}(t) x=u(t ; x)$ is a well-defined linear operator on Banach space $X_{1}=\left(D(A),\|\cdot\|_{A}\right)$ where $\|\cdot\|_{A}$ is the graph norm on $X_{1}$. Before proving the boundedness of $W_{1}(t)$ we notice that $Y=\left(C^{1}\left(I_{T}, X_{1}\right),\|\cdot\|^{\prime}\right)$, where $\|f\|^{\prime}=\|f\|_{\infty}+\sum_{i=1}^{n}\left\|\frac{\partial}{\partial t_{i}} f\right\|_{\infty}$ is a Banach space. Next we show that the mapping $\Phi: X_{1}$ $\rightarrow Y$ defined by $\Phi(x)(t)=W_{1}(t)$ is closed, for; suppose $x_{m} \rightarrow x$ in $X_{1}$ and $\Phi\left(x_{m}\right) \rightarrow f$ in $Y$. Integrating of (7) for initial value $x_{m}$, we have

$$
\begin{aligned}
W_{1}\left(t_{1}, t_{2}, \ldots, t_{n}\right) x_{m}=W_{1}\left(0, t_{2}, \ldots, t_{n}\right) x_{m}-\sum_{i=2}^{n} \int_{0}^{t_{1}} \frac{\partial}{\partial t_{i}} W_{1}\left(s, t_{2}, \ldots, t_{n}\right) x_{m} d s & \\
& +\int_{0}^{t_{1}} A W_{1}\left(s, t_{2}, \ldots, t_{n}\right) x_{m} d s .
\end{aligned}
$$

As $m \rightarrow \infty$ by our choosing of the norm and the closeness of $A$ we get

$$
\left\|\frac{\partial}{\partial t_{i}} W_{1}(\cdot) x_{m}-\frac{\partial}{\partial t_{i}} f(\cdot)\right\|_{\infty} \rightarrow 0, \quad \text { as } m \rightarrow \infty, \quad i=1,2, \ldots, n
$$


and

$$
\begin{aligned}
\left\|W_{1}(\cdot) x_{m}-f(\cdot)\right\|_{\infty} & =\operatorname{Sup}_{t \in I_{T}}\left(\left\|W_{1}(t) x_{m}-f(t)\right\|_{A}\right) \\
& =\operatorname{Sup}_{t \in I_{T}}\left(\left\|W_{1}(t) x_{m}-f(t)\right\|+\left\|A W_{1}(t) x_{m}-A f(t)\right\|\right) \rightarrow 0
\end{aligned}
$$

as $m \rightarrow \infty$. Hence

$$
\begin{aligned}
f\left(t_{1}, t_{2}, \ldots, t_{n}\right)=f\left(0, t_{2}, \ldots, t_{n}\right)-\sum_{i=2}^{n} \int_{0}^{t_{1}} \frac{\partial}{\partial t_{i}} f\left(s, t_{2}, \ldots, t_{n}\right) d s & \\
& +\int_{0}^{t_{1}} A f\left(s, t_{2}, \ldots, t_{n}\right) d s .
\end{aligned}
$$

It gives

$$
\left\{\begin{array}{l}
\sum_{i=1}^{n} \frac{\partial}{\partial t_{i}} f\left(t_{1}, t_{2}, \ldots, t_{n}\right)=A u\left(t_{1}, t_{2}, \ldots, t_{n}\right) \\
f(0)=\lim _{m \rightarrow \infty} W_{1}(0) x_{m}=x .
\end{array}\right.
$$

So $f$ is a solution of (7) and by the uniqueness of solution we conclude $f(t)=W_{1}(t) x$, equivalently $\mathrm{f}$ is closed and by closed graph theorem $\Phi$ is bounded, thus $\operatorname{Sup}_{t \in I_{T}}\left\|W_{1}(t)\right\|$ $<\infty$.

As in Theorem $2.2 W_{1}(t)$ can be extended to a $C_{0}$ - $n$-parameter semigroup $\left(W, \mathbb{R}_{+}^{n}, X_{1}\right)$. Let $\left(H_{1}, H_{2}, \ldots, H_{n}\right)$ be the infinitesimal generator of $W$, for $x \in \bigcap_{i=1}^{n} D\left(H_{i}\right) \subseteq D(A)$ we have

$$
\frac{\partial}{\partial t_{i}} W(t) x=H_{i} W(t) x
$$

Thus

$$
\sum_{i=1}^{n} \frac{\partial}{\partial t_{i}} W(t) x=\left(\sum_{i=1}^{n} H_{i}\right) W(t) x=A W(t) x .
$$

From the continuity of $\frac{\partial}{\partial t_{i}} W(t) x$ and strong continuity of $W(t) x$, the fact that $\sum_{i=1}^{n} H_{i} W(t) x$ $=W(t) \sum_{i=1}^{n} H_{i} x$ (Proposition 1.2), and the closedness of $A$ as $t \rightarrow 0$, the last equality yields

$$
\sum_{i=1}^{n} H_{i}(x)=A(x), \text { for each } x \in \bigcap_{i=1}^{n} D\left(H_{i}\right) .
$$

Applying Lemma 2.4 shows that there is $\omega>0$ such that for each $\lambda, \lambda^{\prime} \geqslant \omega$, we have

$$
R\left(\lambda^{\prime} ; H_{j}\right) R\left(\lambda ; H_{i}\right)=R\left(\lambda ; H_{i}\right) R\left(\lambda^{\prime} ; H_{j}\right)
$$


Now let $H_{1}^{\prime}=H_{1}+I$ and $H_{2}^{\prime}=H_{2}-I$, if $\omega^{\prime}=\omega+1$ and $\lambda, \lambda^{\prime} \geqslant \omega^{\prime}$, we have $\lambda+1, \lambda^{\prime}-1 \geqslant \omega$ and

$$
\begin{aligned}
R\left(\lambda^{\prime} ; H_{1}^{\prime}\right) R\left(\lambda ; H_{2}^{\prime}\right) & =R\left(\lambda^{\prime}-1 ; H_{1}\right) R\left(\lambda+1 ; H_{2}\right) \\
& =R\left(\lambda+1 ; H_{2}\right) R\left(\lambda^{\prime}-1 ; H_{1}\right) \\
& =R\left(\lambda ; H_{2}^{\prime}\right) R\left(\lambda^{\prime} ; H_{1}^{\prime}\right) .
\end{aligned}
$$

Similarly $R\left(\lambda ; H_{i}^{\prime}\right) R\left(\lambda^{\prime} ; H_{j}\right)=R\left(\lambda^{\prime} ; H_{j}\right) R\left(\lambda ; H_{i}^{\prime}\right)$, for $\lambda, \lambda^{\prime} \geqslant \omega^{\prime}, i=1,2$, and $j=3,4, \ldots, n$. By Lemma $2.3 H_{1}^{\prime}$ and $H_{2}^{\prime}$ are the infinitesimal generators of two $C_{0}$-oneparameter semigroups of operators. With the above equalities and Lemma 2.4, this shows that $\left(H_{1}^{\prime}, H_{2}^{\prime}, H_{3}, \ldots, H_{n}\right)$ is the infinitesimal generator of a $C_{0}$ - $n$-parameter semigroup, say $\left(W^{\prime}, \mathbb{R}_{+}^{n}, X_{1}\right)$. So by Lemma 2.3 , for each $x \in X_{1}$,

$$
W^{\prime}\left(t e_{1}\right) x=W\left(t e_{1}\right) x+\int_{0}^{t} W\left((t-\mu) e_{1}\right) W^{\prime}\left(\mu e_{1}\right) x d \mu
$$

and

$$
W^{\prime}\left(t e_{2}\right) x=W\left(t e_{2}\right) x-\int_{0}^{t} W\left((t-\nu) e_{2}\right) W^{\prime}\left(\nu e_{2}\right) x d \nu
$$

Also $W^{\prime}\left(t e_{i}\right)=W\left(t e_{i}\right)$, for $i>2$. We conclude that for $x \in \bigcap_{i=1}^{n} D\left(H_{i}\right)$,

$$
\frac{\partial}{\partial t_{i}} W^{\prime}\left(t_{1}, t_{2}, \ldots, t_{n}\right) x= \begin{cases}H_{i}^{\prime} W^{\prime}\left(t_{1}, t_{2}, \ldots, t_{n}\right) & i=1,2 \\ H_{i} W^{\prime}\left(t_{1}, t_{2}, \ldots, t_{n}\right) & i>2\end{cases}
$$

Hence by (8)

$$
\left\{\begin{array}{l}
\frac{\partial}{\partial t i} W^{\prime}(t)=\left(H_{1}^{\prime}+H_{2}^{\prime}+H_{3}+\cdots+H_{n}\right) W^{\prime}(t)=\sum_{i=1}^{n} H_{i} W^{\prime}(t)=A W^{\prime}(t) x \\
W^{\prime}(0)=x
\end{array}\right.
$$

But the solution of ( 7$)$ is unique, and so for $i=1, \ldots, n$ and $0 \leqslant t \leqslant T_{i}$,

$$
W^{\prime}\left(t e_{i}\right)=W\left(t e_{i}\right)
$$

This implies that

$$
\begin{aligned}
W\left(t e_{1}\right) x=W^{\prime}\left(t e_{1}\right) x & =W\left(t e_{1}\right) x+\int_{0}^{t} W\left((t-\mu) e_{1}\right) W^{\prime}\left(\mu e_{1}\right) x d \mu \\
& =W\left(t e_{1}\right) x+\int_{0}^{t} W\left(t e_{1}\right) x d \mu \\
& =W\left(t e_{1}\right) x+t W\left(t e_{1}\right) x
\end{aligned}
$$

So $t W\left(t e_{1}\right) x=0$ or $W\left(t e_{1}\right) x=0$. This is a contradiction, because $0=\lim _{t \rightarrow 0} W\left(t e_{1}\right) x$ $=x \neq 0$. Thus (7) cannot have a unique solution for each $x \in D(A)$. 
REMARK 2.6. Our technique for proving Theorem 2.2 and a part of Theorem 2.5 is based on Hille's technique for one-parameter case [5]. $C_{0}-n$-parameter semigroups are solutions of many initial value problems contain partial derivative and as in previous Theorem, $C_{0}-n$-parameter semigroups can be used for showing that these initial value problems cannot have a unique solution. As another example for second order initial value problems, consider the two-parameter initial value problem

$$
\left\{\begin{array}{l}
\frac{\partial}{\partial s} \frac{\partial}{\partial t} u(s, t)=A u(s, t) \\
(s, t) \in[0, S] \times[0, T] \\
u(0,0)=x, \quad x \in D(A)
\end{array}\right.
$$

where $A$ is a closed operator. If this problem has a unique solution for each $x \in D(A)$ in both $I_{(S, T)}$ and $I_{\left(S^{\prime}, T^{\prime}\right)}$ for which $I_{\left(S^{\prime}, T^{\prime}\right)} \subseteq I_{(S, T)}$, then $W_{1}(s, t)=u(s, t ; x)$ can be extended to a $C_{0}$-two-parameter semigroup on Banach space $X_{1}=\left(D(A),\|\cdot\|_{A}\right)$, with the infinitesimal generator $(H, K)$. We know $\overline{D(H K) \cap D(K H)}^{1 / \cdot \|_{A}}=D(A)$, (it can be proved completely similarly to the proof of Proposition 1.2 (b)), so $D(H K) \cap D(K H) \neq 0$. Now for $x \in D(H K) \cap D(K H)$, by Proposition $1.2 H K(x)=K H(x)$ and one can see that this is equal to $A(x)$. Also it can be checked that $(H / 2,2 K)$ is the generator of $W^{\prime}(s, t)=W(s / 2,2 t) \neq W(s, t)$. So for $x \in D(H K) \cap D(K H)$,

$$
\left\{\begin{array}{l}
\frac{\partial}{\partial s} \frac{\partial}{\partial t} W^{\prime}(s, t)=\left(\frac{1}{2} H\right)(2 K) W^{\prime}(s, t) x=H K W(s / 2, t) x=A W^{\prime}(s, t) \\
W^{\prime}(0,0) x=x
\end{array}\right.
$$

and this is a contradiction with the uniqueness of solution.

\section{REFERENCES}

[1] P.L. Butzer and H. Berens, Semi-group of operators and approximation (Springer-Verlag, New York, 1967).

[2] K.J. Engle and R. Nagle, One-parameter semigroups for linear evoluation equations (Springer-Verlag, New York, 2000).

[3] A. Friedman, Partial differential equations (Holt, Rinhart and Winston, New York, 1969).

[4] E. Hille, 'On the differentiability of semigroup of operators', Acta Sci. Math. (Szeged) 12 (1950), 19-24.

[5] E. Hille, 'One generalization du problem de Cauchy', Ann. Inst. Fourier 4 (1952).

[6] E. Hille and R.S. Phillips, Functional analysis and semi-groups, Amer. Math. Soc. Colloq. Publ. 31 (American Mathematical Society, Providence, R.I., 1957).

[7] A. Pazy, 'Semigroups of linear operators and applications to partial differential equations', Applied Mathematical Sciences 44 (Springer-Verlag, New York).

[8] R.S. Phillips, 'A note on abstract Cauchy problem', Proc. Nat. Acad. Sci. (1954), 244-248. 
Department of Mathematics

Ferdowsi University of Mashhad

P.O. Box 1159-91775

Masjad

Iran

e-mail: Niknam@math.um.ac.ir

Janfada@math.um.ac.ir 